\title{
XAFS studies of nickel-doped lead telluride
}

\author{
Ivana Radisavljević ${ }^{a, *}$, Nikola Novaković ${ }^{a}$, Nenad Ivanović ${ }^{a}$, Nebojša Romčević ${ }^{b}$, \\ Miodrag Manasijević ${ }^{a}$, Heinz-Eberhard Mahnke ${ }^{c}$ \\ ${ }^{a}$ Vinča Institute of Nuclear Sciences, University of Belgrade, POB 522, 11001 Belgrade, Serbia \\ ${ }^{\mathrm{b}}$ Institute of Physics, University of Belgrade, Pregrevica 118, 11000 Belgrade, Serbia \\ ${ }^{\mathrm{c}}$ Helmholtz-Zentrum Berlin für Materialien und Energie GmbH, D-14109 Berlin, Germany
}

\section{A R T I C L E I N F O}

Keywords:

Lead telluride

Impurities

Defects

XAFS

\begin{abstract}
A B S T R A C T
The problem of impurities and defect states in lead telluride-based semiconductors is of crucial importance for their practical applications. X-ray absorption fine structure (XAFS) techniques are capable to address some of the key issues regarding impurities position, their valent state, as well as the local structural changes of the host lattice in the immediate surrounding of the impurity atoms. In this paper we present the results of the Ni K-absorption edge XAFS studies of Ni-doped PbTe at different temperatures. Analysis of near edge and extended XAFS regions of the measured spectra provided information about exact local environment and lattice ordering around $\mathrm{Ni}$ atoms.
\end{abstract}

(c) 2009 Elsevier B.V. All rights reserved.

\section{Introduction}

Lead telluride-based semiconductors are extensively used in optoelectronics and thermoelectronics [1-4], and recently also in spintronics and quantum electronics [5,6]. Such a wide range of their application is made possible by their unique properties (tunable direct energy gap, high efficiency of radiative recombination, high values of static dielectric constant, small effective mass of carriers, etc.) and versatile possibilities of their combination. Introduction of a particular dopant atom into the host material can lead to appearance of qualitatively new material properties. Whereas group III impurities induce interesting electronic (pinning of the free carriers concentration and Fermi level) and optical properties (strong plasmon-phonon coupling, activation of local optical modes), transition metals act as magnetic impurities and can provoke interesting effects such as giant magnetoresistance. In the case of a large difference between ionic radii and polarizability of the interchanging ions, impurity atoms are readily displaced from the regular lattice positions, creating that way dipoles whose ordering can lead to ferroelectric phase transitions [7,8]. Although a lot of work has been devoted to study defect states in lead telluride-based semiconductors [7-14] they are not yet fully understood. Most theoretical studies [12,13] assume impurities positioned at ideal lattice sites, which often proved not to be the case. Therefore, it is very important to resolve local structures around impurities in order to better understand

\footnotetext{
* Corresponding author. Tel.: +381113408610; fax: +381113440100.

E-mail address: iva@vinca.rs (I. Radisavljević).
}

their influence on macroscopic characteristics of the materials they are incorporated in.

Element specific X-ray absorption fine structure (XAFS) spectroscopy is at present one of the most powerful tools used for studying structural, electronic and magnetic properties of materials. The near edge region of the absorption spectrum (XANES) is sensitive on electronic structure and contains information on oxidation state and the local coordination geometry around the absorbing atom, whereas the high energy region (EXAFS) is associated with the arrangement of atoms and provides information about the short range order in terms of number, type and distances of atoms in coordination shells and their disorder parameters. Using XAFS spectroscopy we have already resolved the local structures in the series of lead telluridebased semiconductors [15-17]. In this work we extend our studies to Ni-doped PbTe, a semimagnetic semiconductor whose electronic and magnetic properties are not completely understood. Lead telluride crystallizes in rocksalt $(\mathrm{NaCl})$-type face-centered-cubic (FCC) structure (space group Fm $3 \mathrm{~m}, a=6.46 \AA$ ), where both $\mathrm{Pb}$ and Te atoms are octahedrally coordinated with atoms of different kind. Nickel forms two types of tellurides: (i) antiferromagnetic semiconductor NiTe with a nickel arsenide (NiAs)-type crystal structure (space group $\mathrm{P}_{3} / \mathrm{mmc}, a=3.390 \AA, c=5.344 \AA$ [18]), where Ni occupy a simple cubic lattice which penetrates a closepacked hexagonal Te lattice, and (ii) paramagnetic metallic $\mathrm{NiTe}_{2}$ with a cadmium iodide ( $\left.\mathrm{CdI}_{2}\right)$-type crystal structure (space group P-3m1, $a=3.861, c=5.297 \AA$ [19]), where Te form a hexagonal close packed arrangement while Ni occupy all octahedral sites in alternate layers. Octahedral $\mathrm{Te}$ coordination of $\mathrm{Ni}$ atoms in both of these tellurides is what provides their partial solubility in PbTe. 


\section{Experiment}

XAFS measurements on PbTe:Ni $\left(n_{\mathrm{Ni}}=1 \times 10^{19} \mathrm{at} / \mathrm{cm}^{3}\right)$ single crystal [20] were performed at A1 beamline of HASYLAB at DESY. The synchrotron radiation source was operating at electron beam energy of $4.5 \mathrm{GeV}$ with a maximum current of $200 \mathrm{~mA}$. Samples were oriented at $45^{\circ}$ to the incident beam and a 7-segment Gedetector was used to collect spectra in fluorescence mode. XANES spectra were modelled with the real space multiple scattering code FEFF8.2 [21] using the self-consistent field approach with Hedin-Lundqvist exchange-correlation. Different atomic arrangements generated by ATOMS package [22] were used as FEFF input files. ATHENA and ARTEMIS packages $[23,24]$ were used to analyze EXAFS data. Fourier transforms (FT) of the $k$-weighted EXAFS function $k \chi(k)$ were performed over the $k$ range from 3 to $13 \AA^{-1}$ using the Hanning windows. The $k_{\min }$ and $k_{\max }$ values were chosen at $\chi(k)$ node points to minimize the spectral broadening, while unphysical peaks in the low $k$-region are suppressed by taking $R_{\mathrm{bkg}}=1.5$. Number of atoms in the $j$ th shell $\left(N_{j}\right)$, mean distance of the $j$ t shell $\left(R_{j}\right)$, and mean-squared displacement of atoms in the $j$ th shell $\left(\sigma^{2}\right)$ were adjusted for each path during the fitting procedure. The same edge shift correction $\left(\Delta E_{0}\right)$ was used for all paths, and amplitude reduction factor $\left(S_{0}^{2}\right)$ was set to 0.7 . Fit-quality factor $R$, estimated by the reduced chi-square $\left(\chi_{v}{ }^{2}\right)$ method as implemented in ARTEMIS, was less than $4 \%$.

\section{Results and discussion}

\subsection{XANES results}

The K-edge XANES spectrum is determined by dipole $1 \mathrm{~s}$ to $4 \mathrm{p}$ transitions, and the rising edge energy position is an approximate measure of the oxidation state of the absorbing atom. Transitions to d-states are formally forbidden but they can occur if there is mixing between $3 \mathrm{~d}$ and $4 \mathrm{p}$ states, in which case they yield structure in the pre-edge region. Appearance of the pre-edge structure is rather common in the transition-metals absorption spectra, though its interpretation is still controversial. It is however known that higher symmetry of the metal site generally leads to weaker pre-edge structure. For the lattice sites with inversion symmetry orbital p-d mixing is not allowed, and preedge features are ascribed to quadrupole s-d transitions.

Fig. 1 shows experimental Ni K-edge XANES spectra recorded at three different temperatures (curves 6-8) and model spectra (curves 1-5) used in their analysis. Experimental spectra are almost identical, which means that the change of Ni immediate surrounding with temperature is insignificant. The similar edge position $\left(E_{0} \approx 8338 \mathrm{eV}\right)$ in all experimental spectra implies that there is no major change of $\mathrm{Ni}$ valent state with rising temperature. The basic XANES model (curve 1) assumes Ni atom replacing $\mathrm{Pb}$ at the ideal $\mathrm{PbTe}$ lattice position. It turned out that there is a large mismatch between experimental and this model spectrum, whose main features remained almost unaffected after allowing the possibility of $\mathrm{Ni}$ atom displacement and also after Te vacancy introduction. The same is true for the model with $\mathrm{Ni}$ atoms in NiTe-like immediate surrounding (curve 2). The $\mathrm{NiTe}_{2}$ model (curve 3 ) brought considerable improvement, although the shape of the main edge is still markedly different from the experimentally observed. The main edge shape slightly changes in the model that combines models 1 and 3 in the ratio 4:2 (curve 4), although it shows tendency to increase in intensity with increasing $\mathrm{NiTe}_{2}$ content. The best description of the main edge part is achieved when Te octahedron is rotated from the $\mathrm{NiTe}_{2}-$ position so to match the orientation that it has in PbTe (curve 5). However, the high energy part of the model spectrum (which is

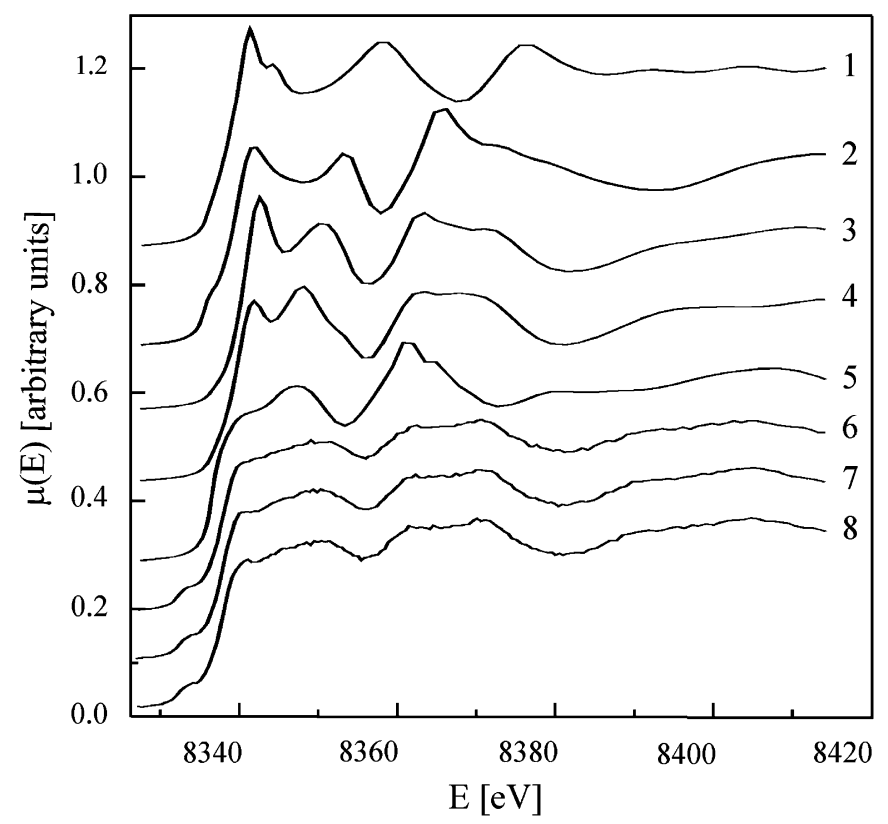

Fig. 1. Ni K-edge experimental XANES spectra (6-8) taken at $20,80 \mathrm{~K}$ and RT, respectively, and theoretical models: $1-\mathrm{Ni}$ replacing $\mathrm{Pb}$ at the ideal PbTe lattice position, $2-\mathrm{Ni}$ as in NiTe, $3-\mathrm{Ni}$ as in $\mathrm{NiTe}_{2}, 4-$ combination of models 1 and 3 in the ratio $4: 2$, and $5-\mathrm{Te}$ octahedron surrounding $\mathrm{Ni}$ rotated from $\mathrm{NiTe}_{2}$ to $\mathrm{PbTe}$ position.

influenced by multiple scattering processes and is therefore very difficult to interpret) is now completely different.

These results indicated very complicated structure of the $\mathrm{Ni}$ immediate surrounding which none of the employed models succeeded to reproduce. The origin of the pre-edge shoulder-like feature is also unclear. The symmetry of Ni sites could be violated due to the Te octahedron deformation, $\mathrm{Ni}$ atoms displacement or presence of Te vacancies. Even if the octahedral symmetry is preserved, the pre-edge structure could appear as a result of quadrupole $s-d$ transitions. The lack of pre-edge structure in the model XANES spectra could be ascribed to limitations of the FEFF based calculations in reproducing near edge region, even though the possibility of quadrupole transition was included. The lack of appropriate reference sample hindered the determination of eventual presence of $\mathrm{Ni}$ atoms in valent states different than expected $2+$.

\subsection{EXAFS results}

Analyses of the EXAFS results confirmed that the local structure around $\mathrm{Ni}$ atoms is completely different from that expected for PbTe and also for NiTe compound. In accordance with XANES analyses, the best fits of the experimental EXAFS spectra are obtained using the $\mathrm{NiTe}_{2}$-structure model. These fits are presented in Fig. 2 and the parameters values are given in Table 1. Tellurium atoms in the first coordination shell are placed at exactly the same distance as in $\mathrm{NiTe}_{2}$. The $\mathrm{Ni}-\mathrm{Te}$ bond is extremely temperature-stable and well defined, as can be deduced from

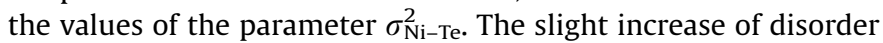
when going from $20 \mathrm{~K}$ to RT can be completely attributed to thermal vibrations, since the bond length remains almost constant with temperature. Second shell is divided into a $\mathrm{Pb}$ and a Ni sub-shell. At low temperatures, three to four $\mathrm{Pb}$ atoms are placed at the distance $\approx 3.71 \AA$, which is $0.15 \AA$ shorter than in $\mathrm{Ni}-\mathrm{Ni}$ distance in $\mathrm{NiTe}_{2}(3.86 \AA$ [19]) and only about $0.5 \AA$ longer than the distance of the first coordination shell in PbTe:Ni $(3.23 \AA$ [20]). One to two $\mathrm{Ni}$ atoms are found at $\approx 3.85 \AA$, the distance 


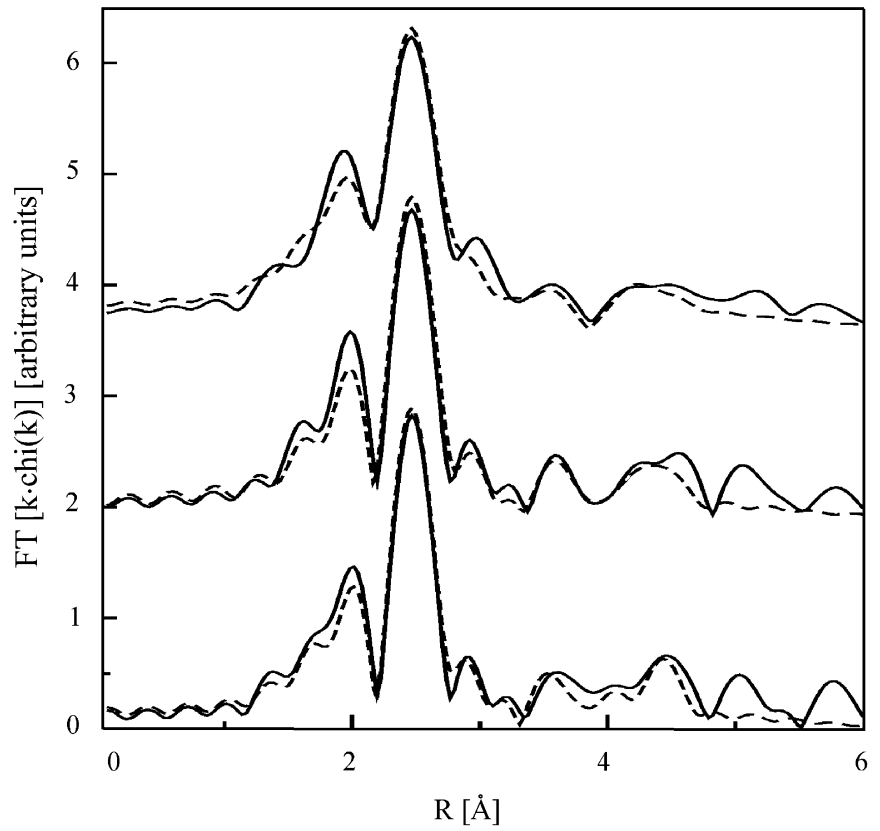

Fig. 2. Fourier transform of the k-weighted Ni K-edge EXAFS spectra taken at RT (upper part) $80 \mathrm{~K}$ (middle part) and $20 \mathrm{~K}$ (lower part). Full line represents experimental data, dashed line represents fit.

Table 1

Structural EXAFS parameters.

\begin{tabular}{lrrr}
\hline & \multicolumn{1}{l}{$20 \mathrm{~K}$} & \multicolumn{1}{c}{$80 \mathrm{~K}$} & \multicolumn{1}{c}{$295 \mathrm{~K}$} \\
\hline$E_{0}(\mathrm{eV})$ & $0.5(6)$ & $0.7(7)$ & $0.6(7)$ \\
$R_{\mathrm{Ni}-\mathrm{Te}}(\AA)$ & $2.580(6)$ & $2.582(6)$ & $2.587(9)$ \\
$\sigma_{\mathrm{Ni}-\mathrm{Te}}^{2}$ & $0.0031(7)$ & $0.0031(8)$ & $0.007(1)$ \\
$N_{\mathrm{Te}}$ & $5.3(4)$ & $5.0(5)$ & $5.3(4)$ \\
$R_{\mathrm{Ni}-\mathrm{Pb}}(\AA)$ & $3.71(4)$ & $3.70(5)$ & $3.72(7)$ \\
$\sigma_{\mathrm{Ni}-\mathrm{Pb}}^{2}$ & $0.004(5)$ & $0.005(8)$ & $0.011(8)$ \\
$N_{\mathrm{Pb}}$ & $3(1)$ & $3(1)$ & $4(1)$ \\
$R_{\mathrm{Ni}-\mathrm{Ni}}(\AA)$ & $3.85(2)$ & $3.85(3)$ & $3.90(6)$ \\
$N_{\mathrm{Ni}}$ & 3 & 3 & 2 \\
$R_{\mathrm{Ni}-\mathrm{Te} 2}(\AA)$ & $4.51(2)$ & $4.52(3)$ & $4.58(7)$ \\
$\sigma_{\mathrm{Ni}-\mathrm{Te} 2}^{2}$ & $0.006(3)$ & $0.008(4)$ & $0.02(1)$ \\
\hline
\end{tabular}

which is again the same as in $\mathrm{NiTe}_{2}$. At $\mathrm{RT}$ both $\mathrm{Ni}-\mathrm{Pb}$ and $\mathrm{Ni}-\mathrm{Ni}$ bonds elongate and their disorder significantly increases. At low temperatures we were even able to locate Te atoms in the third coordination shell at distance which is slightly shorter than corresponding distance in $\mathrm{NiTe}_{2}(4.55 \AA$ [19]) and the distance of

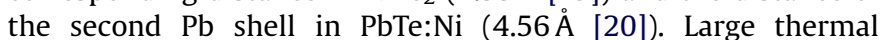
vibrations and probably also larger statistical disorder influence precise determination of the $\mathrm{Ni}-\mathrm{Te}_{2}$ distance at $\mathrm{RT}$ and the exact number of Te atoms in this shell (which was therefore fixed to expected six). These results could be indication of limited solubility of $\mathrm{NiTe}_{2}$ in PbTe. However, according to our preliminary analysis of the EXAFS results obtained at $\mathrm{Pb} \mathrm{L}_{\mathrm{III}}$-edge at $20 \mathrm{~K}, \mathrm{~Pb}$ sub-lattice is extremely stable and remains almost unaffected by the presence of $\mathrm{Ni}$ at this doping level. All the parameters up to the third shell, in consistency with XRD measurements [20], correspond to the NaCl-type structure. Despite the fact that there are no other crystalline phases in the sample, local structure around $\mathrm{Ni}$ atoms is to a large extent $\mathrm{NiTe}_{2}$-alike as a result of stronger and predominately covalent $\mathrm{Ni}$-Te bonding. Even though long-range Coulomb interaction is expected to be strongly screened in PbTe compound due to the large dielectric constant, the $\mathrm{Ni}-\mathrm{Ni}$ interaction is still strong enough to bring $\mathrm{Ni}$ atoms close to each other, even at this small concentration. Similar situation is observed in PbTe:Mn, only the transformation of the $\mathrm{Mn}$ immediate surrounding to MnTe was not completed which resulted in $\mathrm{Mn}$ off-centering from the regular $\mathrm{PbTe}$ lattice position [15,17]. Definite answers about Ni doping effects on $\mathrm{PbTe}$ will be possible to obtain after completing the Te K-edge data analysis.

In conclusion, we have studied nickel-doped PbTe by means of Ni K-absorption edge XAFS measurements at different temperatures. Detailed analysis of the XANES and EXAFS regions of the absorption spectra revealed that the local structure around $\mathrm{Ni}$ atoms strongly deviates from the host $\mathrm{PbTe}(\mathrm{NaCl})$ structure, and that it is very close to the $\mathrm{CdI}_{2}$-type structure of $\mathrm{NiTe}_{2}$ compound. Tellurium atoms in the immediate $\mathrm{Ni}$ surrounding form a temperature-stable octahedron whose orientation is adjusted to PbTe long-range order. The second, and even the third shell are also more $\mathrm{NiTe}_{2}$ structure-alike. However, due to strong Coulomb screening and low $\mathrm{Ni}$ concentration, influence of $\mathrm{Ni}$ doping is localized and the long-range order is well preserved.

\section{Acknowledgements}

The authors gratefully acknowledge HASYLAB for providing beamtime and A1 beamline scientists Dr. E. Welter and Dr. A. Webb for their assistance during XAFS measurements. This work was supported by Serbian Ministry of Science and Technological Development under the Grant 141009.

\section{References}

[1] M.S. Dresselhaus, G. Dresselhaus, X. Sun, Z. Zhang, S.B. Cronin, T. Koda J.Y. Ying, Microscale Thermophysical Eng. 3 (1999) 89.

[2] J.H. Dughaish, Physica B 322 (2002) 205.

[3] K.F. Hsu, S. Loo, F. Guo, W. Chen, J.S. Dyck, C. Uher, T. Hogan, E.K. Polychroniadis, M.G. Kanatzidis, Science 303 (2004) 818

[4] T.C. Harman, P.J. Taylor, M.P. Walsh, B.E. LaForge, Science 297 (2002) 2229.

[5] G. Grabecki, Mater. Sci.-Poland 24 (2006) 591.

[6] E. Kaufmann, G. Springholz, T. Schwarzl, G. Hesser, F. Schäffler, W. Heiss, K. Koike, T. Ikatura, T. Hotei, M. Yano, T. Wojtowicz, J. Phys.: Condens. Matter 20 (2008) 454216.

[7] Q.T. Islam, B.A. Bunker, Phys. Rev. Lett. 59 (1987) 2701

[8] A.I. Lebedev, I.A. Sluchinskaya, Ferroelectrics 157 (1994) 275

[9] D.T. Morelli, J.P. Heremans, C.M. Thrush, Phys. Rev. B 67 (2003) 035206.

[10] L.I. Rabova, D.R. Khokhlov, JETP Lett. 80 (2004) 133.

[11] A.I. Lebedev, I.A. Sluchinskaya, S.G. Nikitenko, S.G. Dorofeev, Phys. Scr. T 115 (2005) 365.

[12] S. Ahmad, S.D. Mahanti, K. Hoang, M.G. Kanatzidis, Phys. Rev. B 74 (2006) 155205.

[13] S. Ahmad, K. Hoang, S.D. Mahanti, Phys. Rev. Lett. 96 (2006) 056403.

[14] R. Saravanan, M. Charles Robert, J. Phys. Chem. Solids 70 (2009) 159

[15] I. Radisavljević, N. Ivanović, N. Novaković, N. Romčević, H.-E. Mahnke, X-ray Spectrom. 36 (2007) 150.

[16] I. Radisavljević, N. Ivanović, N. Novaković, N. Romčević, H.-E. Mahnke, AIP Conf. Proc. 899 (2007) 642.

[17] I. Radisavljević, N. Ivanović, N. Novaković, M. Manasijević, N. Romčević H.-E. Mahnke, M. Mitrić, J. Phys.: Condens. Matter, submitted.

[18] Y.P. Yadava, R.A. Singh, J. Mater. Sci. Lett. 4 (1985) 1421.

[19] F. Hulliger, Structural chemistry of layer type phases, in: F. Levy (Ed.), Physics and Chemistry of Materials with Layered Structures, Vol. 5, Reidel, Dordrecht 1976.

[20] N. Romčević, J. Trajić, T.A. Kuznetsova, M. Romčević, B. Hadžić, D.R. Khokhlov, J. Alloys Compd. 442 (2007) 324.

[21] A.L. Ankudinov, C. Bouldin, J.J. Rehr, J. Sims, H. Hung, Phys. Rev. B 65 (2002) 104107.

[22] B. Ravel, J. Synchrotron Rad. 8 (2001) 314

[23] M. Newville, J. Synchrotron Rad. 8 (2001) 322

[24] B. Ravel, M. Newville, J. Synchrotron Rad. 12 (2005) 537. 